\title{
Realization and Research of Intelligent system of client electricity information based on the Big Data Processing Technology
}

\author{
Wen Ming-xin ${ }^{1, a}$ Chu Ming-hua ${ }^{1, b}$ Fang Lei ${ }^{1, c}$ Chen Wen-jiao ${ }^{1, d}$ \\ 1. State Grid Weifang Electric Power Supply Company Weifang 261021, China \\ a1014554345@qq.com, bsamchu2011@vip.sina.com, ${ }^{\mathrm{c}} 13606365728 @ 163 . c o m$
}

\begin{abstract}
Keywords: Big data, Data collection, Power information system, Software design, Intelligent clustering subsystem
\end{abstract}

Abstract. Big data is the focus in power system currently. In order to analyze and classify the electricity model of clients, identify the avoiding peak space intelligently, extract value-added information of clients and control the electric load actively, it is extremely necessary to analyze the information of electricity clients and establish an intelligent system for electricity client information based on big data processing technology. A data collection method on client electricity information is proposed in this paper, which could be a security of client's electricity information. In order to improve the quality of data collection so as to consolidate the reliability of system information, distributed data processing platform based on Hadoop is designed to collect and process information of clients. On the basis of which, a software of intelligent system of client electricity information is designed as well, which consists of a series of intelligent clustering subsystems. The research results provide theoretical guidance for the application of big data processing technology in power system.

\section{Introduction}

With the rapid development and wide application of the Internet, cloud storage and Internet of things, big data has become the focus of research currently [1]. The competitiveness and attractiveness of client's demand side would be promoted when big data technology is applied in intelligent system of power grid [2], analysis tools and decision support would be provided meanwhile for the healthy operation and sustainable development of Power Grid Corp [3]. However, lack of theoretical guidance on big data processing technique in power industry and application system of power industry based on big data make it imminent to employ big data technology in power industry. In order to analyze and classify the electricity model of clients, identify the avoiding peak space intelligently, extract value-added information of clients and control the electric load actively, it is extremely necessary to analyze the information of electricity clients and establish an intelligent system for electricity client information based on big data processing technology.

Intelligent system of client electricity information based on big data processing technology is proposed in this paper. On the basis of intelligent system, software is designed to deeply analyze the information of electricity clients, that is, to analyze and classify the electricity model of clients, to identify the avoiding peak space intelligently, to extract value-added information of clients and to control the electric load actively. The research results provide theoretical guidance for the application of big data processing technology in power system.

\section{Design of data processing system on electricity information}

The most basic and significant function of client intelligent information system is data collection and processing, that is, data processing is the basis of other system functions.

The data collection management process is shown in Fig.1. 


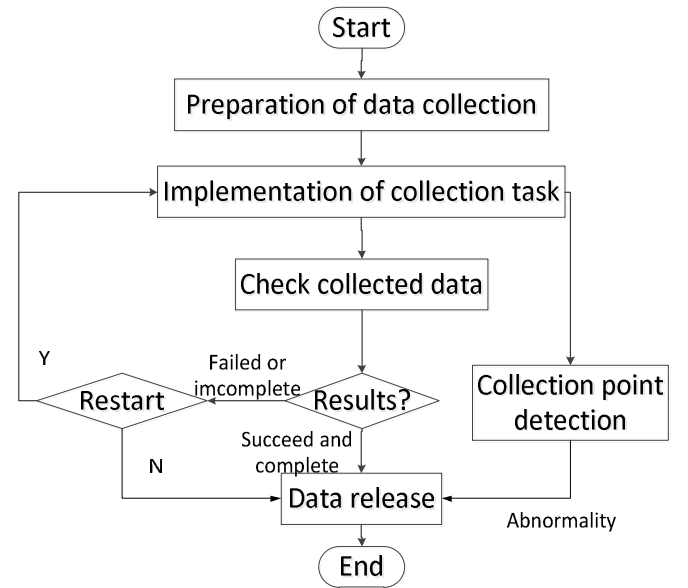

Fig.1 The data collection management process

The preparation of data collection task is required firstly. The collection task is implemented in the following step. Then, the quality of collected data is checked according to certain rules, if data collection is succeeded and collected data is complete, the qualified data is to release. Otherwise, the data collection task is needed to restart. During the data collection processing, the system detects the collected points in real time. Abnormal information is released immediately when an abnormality is detected. High reliability is indicated in this method and data collection efficiency is improved at the same time.

The analysis and evaluation of big data processing platform are carried out within several aspects, such as performance, cost, security, implementation difficulty and application. A Hadoop big data processing platform appropriate for electrical client information and load control is established, as is shown in Fig.2, according to the comparison.

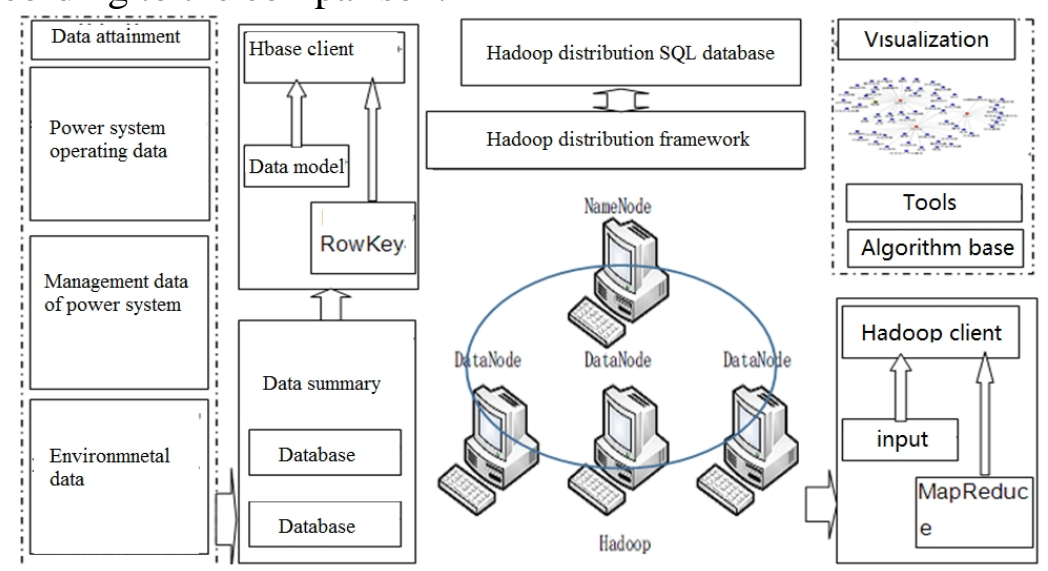

Fig.2 Distributed data processing platform based on Hadoop

The collected electricity information data are firstly summed up and integrated, according to platform frame shown in Fig.2, the characteristics of different types of data are extracted so as to establish the data rules, and the relationship between the data mapping and model establishment is realized subsequently. On the basis of which, with the utilization of HBASE database, the corresponding data is stored in column. Then, through the RowKey generator, based on Hadoop distributed architecture, the unified and distributed data storage, retrieval and management of the data are realized. In the data retrieval and output process, the MapReduce key value relationship is utilized to achieve distributed data parallel processing, at the same time, analysis and processing on data are realized through the data association rules, data clustering, classification, prediction and machine learning algorithm. Finally, the data visualization is displayed by visual software tools.

The specified calculation model can be selected at the interface to analyze the corresponding data, then select the data attributes that need to be calculated in the metadata. Then the calculation model, calculation attributes and metadata are transferred to the service semantic layer and the semantic of algorithm on specified calculation is formed in the service semantic layer meanwhile. The semantic of 
algorithm will be converted into fixed calculation SQL statements and the SQL statements are transferred into computational scripts and computational tasks Job subsequently. The specified calculation model is selected to implement computational scripts according to the definition of Job, and the results is output to the specified result table according to defined result model. Therefore, electricity information retrieval could be realized in data service.

\section{The software design of the intelligent electric information system}

Schematic diagram of software frame concerning the intelligent system of client electricity information, as shown in Fig.3, which is based on big data progressing technology.

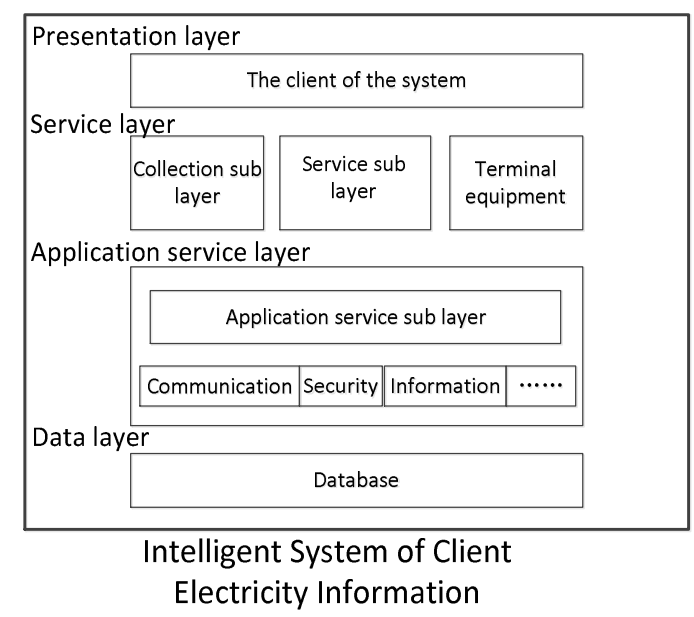

Fig.3 Diagram of intelligent system of client electrical information data

Distributed multi-tier architecture is employed in electricity information intelligent system, the software of which consists of presentation layer, service layer, application service layer and data layer. Power grid real time load analysis module, client power mode analysis module, client value added information analysis module and client load avoiding peak analysis module are designed according to requirements, aiming at further analyzing client electricity information.

Presentation layer can be regarded as the client of system, that is, the interface of intelligent system for client electricity information. The function of presentation layer is to realize the communication between business operator and service layer. All the input data can be acquired through presentation layer, then transmitted to the service layer, and the data and processing results from the service layer will be received and displayed at the same time.

The service layer is responsible for handing control instructions and requests, and making a great quantity of business rules and processing logic achieved. Service layer is considered as the processing section in core business of the whole system, that is, to feedback the results and information to the operator in time. According to the application characteristics of the system, the service layer can be divided into the collection sub layer and the service sub layer.

In the intelligent system of client electricity information, various types of terminal equipment can be connected with the collection sub layer through various communication methods, so as to execute the tasks and control commands of service sub layer. The collection sub layer could acquire the settings of terminal parameters, collect terminal data and analyze data subsequently. The service function of electricity information collection system can be achieved when the technical means provided by application service layer is utilized by the corresponding service sub layer. In order to further analyze client electricity information, client power mode analysis module, client value added information analysis module and client load avoiding peak analysis module comprise the service sub layer collectively.

Global universal information and security and communication components are mainly provided by the application service layer, so as to realize service sub layer specialized for electricity 
information system. General technical support in service layer is provided by application service layer to ensure the reliable and efficient communication capability of electrical information system, and to meet the requirement of concurrent access to the application. The reliable interaction between components can be achieved in the application service layer, moreover, this layer can be easily extended.

\section{Analysis on client power mode and application of intelligent clustering subsystem}

Power client electricity mode analysis and clustering subsystem are proposed based on key characteristics of electricity clients in power industry. Cluster analysis on massive client load can be analyzed through the proposed clustering subsystem.

Intelligent mining on avoiding peak space can be achieved through client load avoiding peak space mining subsystem. The provided avoiding peak space at different temperature setting points can be identified intelligently according to the load of air conditioning and heat pump in public organizations and commercial buildings. As to industrial client's load, the relationship between the load change and the combination, the start/stop status can be mined at first, the industrial client avoiding load space can be identified in accordance with the relationship subsequently.

Client value added information mining subsystem provides value added information service for electrical clients, government agencies and financial institutions. On the basis of the development trend of client history data, the industry boom index of clients can be mined through this subsystem, and industry boom forecast service could be provided for the clients. Combing national economy industrial boom index with industrial chain effect, the developing trend of national economy and industrial structure change characteristics are predicted and extracted, finally, national economic development trend prediction and the change of industrial structure analysis are provided by government agencies and financial institutions.

In the client load control subsystem, applicable control strategy can be designed to control client load flexibly and enhance the active control of the power grid to clients, according to different types of clients, such as public institutions, commercial buildings, factories, and so on.

\section{Conclusions}

This paper proposes a method of data collection to ensure the security and reliability of client electricity information data, which features high reliability and high efficiency. Meanwhile, a distributed data processing platform based on Hadoop is raised for acquiring and processing client electricity information data. On the basis of which, the Intelligent system of client electricity information based on big data processing technology is built. Finally, the software of intelligent client electricity information system is designed to model a series of intelligent clustering subsystems, such as client power model analysis clustering subsystem, intelligent identification on avoiding peak space system, client value added information mining system and client load active control subsystem. The research results provide theoretical guidance for the application of big data processing technology in power system.

\section{References}

[1]Dongxia Zhang, Xin Miao, Liping Liu, et al. Research on the development of big data technology in Smart Grid[J]. Proceedings of CSEE, 2015, 35(1): 2-12 (in Chinese).

[2]Xiaosheng Peng, Diyuan Deng, Shijie Cheng, et al. Key technologies of power big data for smart grid application[J]. Proceedings of CSEE, 2015, 35(3): 503-511 (in Chinese).

[3]Xiang Huang, Zhigang Chen. Research on big data information platform of smart grid[J]. Southern Energy Construction, 2015, 2(1): 17-21 (in Chinese). 